\title{
The magic continues for the iPS strategy
}

\author{
Duanqing Pei ${ }^{1}$
}

${ }^{\prime}$ Stem Cell and Cancer Biology Group, Laboratory of Regenerative Biology, South China Institute for Stem Cell Biology and Regenerative Medicine, Guangzhou Institute of Biomedicine and Health, Chinese Academy of Sciences, Guangzhou 510663, China. pei_duanqing@gibh.ac.cn

Cell Research (2008) 18:221-223. doi: 10.1038/cr.2008.21; published online 4 February 2008

Breathtaking progresses have been made since the first report from Yamanaka and colleagues on the reprogramming of mouse fibroblasts into pluripotent stem cells by defined factors in 2006 [1, 2]. First, several groups have independently reported the derivation of pluripotent stem cells from mouse somatic cells by Nanogor Oct4- based selection strategies and demonstrated that iPS cells possess almost identical properties as ES cells [3-5]. Second, iPS cells can be generated without drug selection, implying that the iPS technology can be applied to any unmodified cells [6-8]. Third, human iPS cells were reported by three independent groups, surprisingly employing different combination of factors including Oct4, Sox2, Myc, Klf4, Nanog, Lin28, SV40L-T, and HTERT [9-11]. Finally, successful treatment of mouse sickle cell anemia by iPS cells derived from autologous skin was reported, paving the way for the application of iPS technology in disease therapies [12]. These progresses were reported in a period of less than 6 months, further demonstrating the intense interest that the iPS technology has generated and the great opportunities it has provided. These rapid progresses foresee an accelerated pace of development for the iPS technology in the near future. Here I review some of the recent breakthroughs and discuss their impact on future developments, especially the potential application in disease therapy.

Drug-free iPS: The original iPS protocol contains two undesirable features from a therapeutic point of view: the need for a selection marker for iPS cells and the retroviral delivery

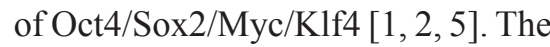
selection markers such as Fbx15-, Nanog- or Oct4- neo ${ }^{\mathrm{r}}$ or puror ${ }^{\mathrm{r}}$ cassettes were engineered into the genome of mouse fibroblasts to confer a selective advantage for iPS clones in the presence of G418 or puromycin [1, 3-5]. However, most of the cells we would like to reprogram, such as those from patients, do not contain such selection markers, thus, may not be suitable for the original iPS protocol. To circumvent this problem, three groups attempted to generate iPS clones without drug selection and succeeded in obtaining iPS cell lines indistinguishable from G418 selected clones [6-8]. The trick lies in the acquired growth advantage of cells infected with the 4 transcription factors over the background MEF cells and the distinct ES-like morphology of the resulting iPS cells [6-8]. In fact, iPS protocol without selection appeared to yield around $10 \times$ more iPS clones [6-8], perhaps, consistent with the notion that the reprogramming process is slow and gradual and early application of drug selection might have eliminated $90 \%$ of potential iPS clones. The development of selectionfree iPS protocol thus brought this technology one step closer towards patient-specific pluripotent cells for transplantation therapy.

Human iPS cells: Many thought that it may take a couple of years to translate the knowledge learnt in mouse to human cells, considering the lack of human cells with similar selection markers [1-3, 5]. It came as a surprise when the Yamanaka group at Kyoto University and Thomson group in Wisconsin published independently the successful generation of human iPS cells from various somatic cells $[9,10]$. Yu from the Thomson group took advantage of a human ES cell line with the Oct4-neo knocked in, similar to the selection system used in mouse $[4,9]$. Through this unique selection system, $\mathrm{Yu}$ and colleagues identified 4 factors out of 14 candidates to be sufficient for the induction of iPS cells from ES-derived CD45+ hematopoietic cells [9]. Surprisingly, instead of Oct4/Sox $2 / \mathrm{Myc} / \mathrm{Klf} 4$ used in mouse, a new combination, Oct4/ Sox $2 / \mathrm{Nanog} / \operatorname{Lin} 28$, was discovered for the generation of human iPS cells [9]. These 4 factors were subsequently shown to be sufficient in reprogramming the unmodified human somatic cell line IMR90 and human newborn foreskin fibroblasts [9]. Based on this observation, one might have thought that the reprogramming of human 
somatic cells must be different from mouse cells. Yet, the Yamanaka group used the same Oct4/Sox2/Myc/Klf4 combination that worked for mouse cells to reprogram human dermal fibroblasts [HDF], after the introduction of a mouse retroviral receptor S1c7a1 to these HDF cells for enhanced transduction efficiency [10]. Based on microarray analysis and characterization of epigenetic markers, these factors appear to exert similar reprogramming effects on somatic cells of human and mouse origins [10]. These two independent studies in fact suggest that there could be more factors potentially important for the reprogramming process. Indeed, Daley and colleagues reported afterwards that hTERT and SV40 large T can enhance the reprogramming efficiency of Oct $4 / \mathrm{Sox} 2 / \mathrm{Myc} / \mathrm{K} 1 \mathrm{f} 4$ on human somatic cells [11]. Thus, it is likely that more factors may be discovered to possess reprogramming potentials as the newly identified Nanog, Lin28, hTERT and SV40 large T.

iPS therapy for mouse: Another exciting result is the first successful therapy demonstrated for iPS cells in mice [12]. Using a humanized knockin mouse model for sickle cell anemia, Jaenisch and colleagues generated iPS cells from these knock-in mice carrying the human $A \gamma$ and $\beta$ s globin genes [12]. Through homologous recombination, the harmful bs allele was subsequently replaced by $\beta A$ allele in iPS cells [12]. The corrected iPS cells were then induced to differentiate into hematopoietic progenitors [HPs] and transplanted back to the sick mice. The HPs derived from $\beta$ A-corrected iPS cells were able to contribute $70 \%$ of the peripheral blood and mice were effectively cured of sickle cell anemia [12]. The safety concern of the myc oncogene was cleverly addressed by cre-mediated excision of the integrated myc-carrying viral genome from the iPS cells [12]. This post-iPS viral excision strategy may prove useful in the eventual application of iPS cells in human therapies.

Into the future: The iPS technology is less than 2 years old, yet exhibited unprecedented promises for both basic research and therapeutic applications. First, one may argue that iPS cells hold the key to our understanding of nuclear reprogramming. As iPS cells are functionally equivalent to ES cells $[1,4,5]$, the process of their induction would be one of the best models to investigate the

A
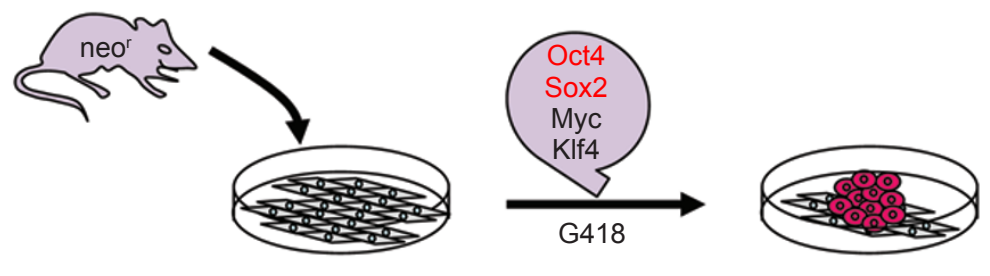

B
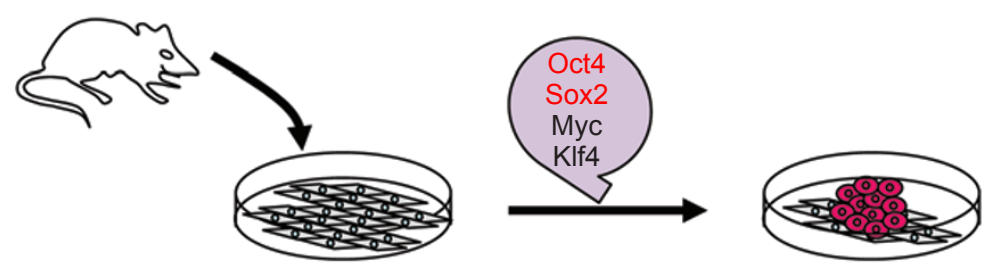

C
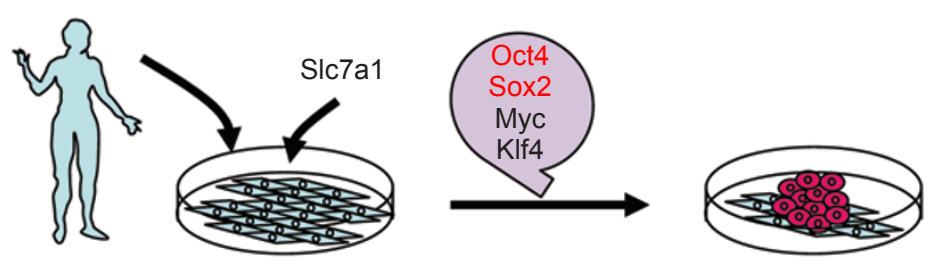

D
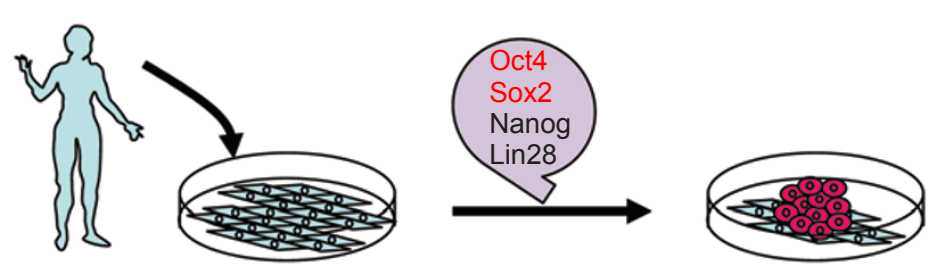

$E$
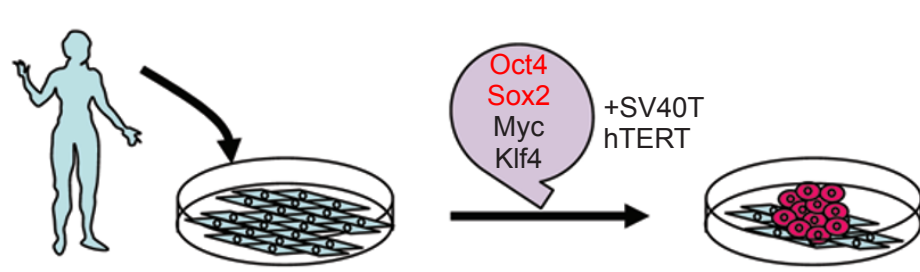

molecular mechanisms of reprogramming. Prior to iPS, the only known way to reprogram somatic nuclei is through SCNT (somatic cell nuclear transfer) [13]. SCNT will remain the standard in the generation of cloned research, thus, may not be readily replaced by iPS. However, SCNT is a technology practiced by only a few labs equipped with expensive equipments and staffed by investigators with years of experiences in this art.

Figure 1 Summary of iPS strategies. A, iPS with selection [references 1, 3-5]; B, iPS without selection [references 6-8]; C, human iPS with mouse retroviral receptor transduction [reference 10]; D and E, human iPS using different combination of factors than mouse iPS [references 9,11 ]

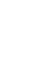


Furthermore, the scarcity of SCNT embryos precludes detailed analysis of the reprogramming process at the cellular and molecular levels. On the contrary, the viral iPS protocol can be easily adapted in standard molecular/cell biology laboratories. Junior scientists such as graduate students or postdocs can be trained to practice iPS in a short period of time. Cells involved in the iPS protocol are abundant enough to allow detailed analyses at the cellular and molecular levels routinely. Therefore, one would argue that the epigenetic reprogramming of somatic nuclei will be dissected quite thoroughly by using the iPS model for both mouse and human cells in the near future. Therapeutically, the iPS technology remains unsuitable for human diseases at the present, due to safety concerns associated with viral transduction of those potentially oncogenic factors [12]. Therefore, one may argue that we are at the beginning of the first generation of iPS technology, i.e., the virally induced PS or ViPS. Future efforts should be focused on identifying alternatives to the viral delivery system and reprogramming factors. Since these factors, Oct4/Sox2/Myc/Klf4, represent definable signaling pathways, it is rational to propose a chemical approach to switch on these pathways for iPS reprogramming. To this end, the nascent discipline of chemical biology may adopt iPS as a worthy field and provide small-molecule tools to regulate the iPS process. One can speculate that somatic cells may eventually be reprogrammed chemically to a pluripotent state. These chemically induced pluripotent stem cells or CiPS cells could be suitable for human therapeutic applications in the future.

\section{References}

1 Takahashi K, Yamanaka S. Induction of pluripotent stem cells from mouse embryonic and adult fibroblast cultures by defined factors. Cell 2006; 126:663676.

2 Qi H, Pei D. The magic of four: induction of pluripotent stem cells from somatic cells by Oct4, Sox2, Myc and Klf4. Cell research 2007; 17:578-580.

3 Maherali N, Sridharan R, Xie W, et al. Directly Reprogrammed Fibroblasts Show Global Epigenetic Remodeling and Widespread Tissue Contribution. Cell Stem Cell 2007; 1:55-70.

4 Okita K, Ichisaka T, Yamanaka S. Generation of germline-competent induced pluripotent stem cells. Nature 2007; 448:313-317.

5 Wernig M, Meissner A, Foreman R, et al. In vitro reprogramming of fibroblasts into a pluripotent ES-cell-like state. Nature 2007; 448:318-24

6 Qin D, Li W, Zhang J, Pei D. Direct generation of ES-like cells from unmodified mouse embryonic fibroblasts by Oct4/Sox2/Myc/Klf4. Cell Res 2007 ; 17:959-962.

7 Meissner A, Wernig M, Jaenisch R. Direct reprogramming of genetically unmodified fibroblasts into pluripotent stem cells. Nat Biotechnol 2007; 25:1177-81

8 Blelloch R, Venere M, Yen J, Ramalho-Santos M. Generation of Induced Pluripotent Stem Cells in the Absence of Drug Selection. Cell Stem Cell 2007; 1: 245-247.

9 Yu J, Vodyanik MA, Smuga-Otto K, et al. Induced pluripotent stem cell lines derived from human somatic cells. Science 2007; 318:1917-1920.

10 Takahashi K, Tanabe K, Ohnuki M, et al. Induction of pluripotent stem cells from adult human fibroblasts by defined factors. Cell 2007; 131:861-872.

11 Park IH, Zhao R, West JA, et al. Reprogramming of human somatic cells to pluripotency with defined factors. Nature 2008; 451:141-146.

12 Hanna J, Wernig M, Markoulaki S, et al. Treatment of sickle cell anemia mouse model with iPS cells generated from autologous skin. Science 2007; 318:1920-1923,

13 Wilmut I, Schnieke AE, McWhir J, Kind AJ, Campbell KH. Viable offspring derived from fetal and adult mammalian cells. Nature 1997; 385:810-813. 